\title{
EL SALVADOR: AHORRO, EXPORTACIONES Y GUERRA
}

\author{
Alvaro Trigueros*
}

\section{Introducción}

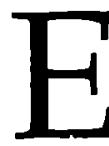

sta ampliamente aceptado que un crecimiento sostenido en la acumulación de capital, y por ende el crecimiento econó mico, es una condición necesaria para el desarrollo económico. También es conocido por definiciones de contabilidad nacional que las inversiones son financiadas por ahorros nacionales, entradas extranjeras o ahorro extranjero, dado que el ahorro extranjero es una variable fuera de control de quienes elaboran las políticas, es importante tener un mejor entendimiento del comportamiento del ahorro nacional. Es de interés para los que elaboran la política saber que grupos tienen más capacidad para ahorrar en orden de proveer con los incentivos apropiados el incremento del ahorro nacional, y dirigir estos fondos a proyectos de inversión.

Este trabajo sostiene que en El Salvador, durante el período 1958-1991, la principal fuente de ahorro nacional son los ingresos generados por el sector exportador, en contra del punto de vista general que trata enteramente de incrementar ingresos o el producto nacional bruto.

La motivación de este estudio fue originada en parte por la observación del gráfico de exportaciones y ahorros contra tiempo. Como podemos ver en la figura 1 ambas variables tienen un desarrollo casi idéntico. Lo cual implica que el coeficiente de correla-

\footnotetext{
* Catedrático del Departamento de Economía de la UCA.
} 
ción simple es 0.812 sugiriendo que tienen una relación especial. Otra motivación fue que en E. S., la política económica, durante el período estudiado ha sido siempre influenciada por un modelo de exportación dirigida, principalmente en favor de exportaciones de productos primarios, café, algodón y caña de azúcar, y secundariamente en favor de productos de consumo final, como resultado, los grupos de empresarios asociados con estos sectores les han proporcionado bajas tasas de impuestos, acceso fácil y barato al mercado de crédito y una infraestructura favorable (Bulmer-Thomas, 1987), y Plupessy (1991), hechos que también comprobaron que podría haber una relación entre ahorro y exportaciones.

Fig. 1: Ahorro Nacional y Exportaciones 1958-1991 (Miles de colones)

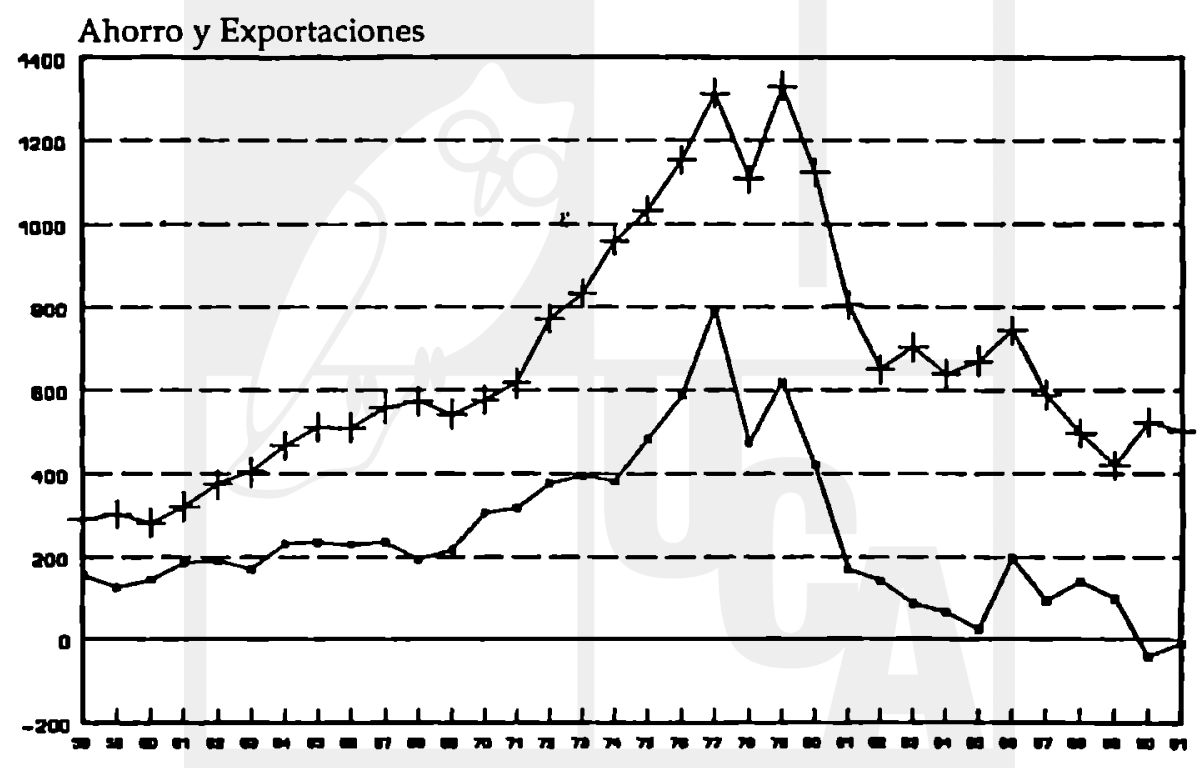

Años

--_ Ahorro Nacional

+ Exportaciones 
La tesis esta dividida en 2 capitulos. El primer capitulo presenta una revisión de la literatura que relaciona los ahorros y las exportaciones, así como los descubrimientos empíricos acerca del tema, y algunas estimaciones de funciones de ahorro para E. S. El segundo capítulo contiene un análisis para determinar que espacio predomina en E.S. y también presenta en el contexto de diferentes modelos, una serie de pruebas mal especificadas comparando el desenvolvimiento de PNB y exportaciones como variables alternativas para explicar el comportamiento del ahorro, concluyendo que la variable más apropiada es el ingreso derivado del sector exportador.

\section{Nota}

A unque el crecimiento sostenido en la acumulación de capital económico, no es considerado condición suficiente, hay otros factores, como el tipo de tecnología adoptada, la igualdad en la distribución, la situación política, la salud y la educación, etc que deben ser tomados en cuenta para evaluar el nivel de desarrollo. Como resultado, el enfoque de este estudio es más difícil de entender.

\section{Capítulo I}

\section{Relación entre ahorro y exportaciones}

Fista sección resume los descubrimientos en la literatura econó primera parte se proporciona el soporte teórico. La segunda parte presenta los resultados empíricos. La sección también muestra en la tercera parte, algunas funciones de ahorro que han sido estimadas para El Salvador.

\section{Ahorro y exportaciones en la teoría económica}

F n el contexto de economía de exportación primaria, donde el nerado por este sector puede jugar un papel muy importante como una fuente de ahorro nacional. Básicamente hay tres puntos de vista que sostienen esta idea. Esta es un simple argumento de la pro- 
pensión marginal a consumir que ha sido aplicado especialmente en el contexto de la estimación de la función de,ahorro ex-ante en modelo de dos brechas. El segundo, es un desarrollo estructuralista (también en un contexto de dos brechas) que toma en cuenta la insuficiencia de intercambio con el extranjero y la ausencia o el reducido tamaño del sector de bienes de capital en la economía. Y el tercero, es un argumento de hipotético ingreso permanente que no ha sido explotado para el caso de ingresos generados por el sector exportador.

Para el primer tipo de argumento, la relación explícita entre el nivel de ingreso en el sector exportador y el ahorro nacional que fue primeramente señalado por Maizels (1968). El quería demostrar que en el campo nacional de ingreso-ahorro, la función de ahorro ex-ante no será estable, pero puede variar de un año a otro. Eso es en la función de ahorro Keynesiana simple.

$$
\mathrm{St}=\mathrm{a}+\mathrm{bYt}
$$

donde St: es el ahorro nacional bruto y $Y t$ es el producto nacional bruto: y el intercepto a: cambiará. La razón para ello es que la propensión marginal a ahorrar PMgS, del ingreso del sector exportador, es más grande que la $\mathrm{PMgS}$ del ingreso de un sector no exportador.

Maizels (1968), encuentra tres razones para justificar su diferenciación entre las dos PMgS: "porque la propensión a ahorrar es más alta en el sector exportador que en cualquier otro o porque los ahorros del gobierno están apoyados en su mayoría por los impuestos al comercio exterior". (p. 58) o porque para un período de tiempo dado un crecimiento sostenido en las exportaciones, podría resultar en un crecimiento en las propensiones de ahorro marginal también en otros sectores.

Lee (1971), se dio cuenta que estas tres razones son debidas a efectos directos de las exportaciones sobre el comportamiento del ahorro. En añade tres efectos indirectos para apoyar la hipótesis de Maizels:

"1. Una asignación más eficiente de recursos inducida por un incremento en las oportunidades de comercio, tal como sugeriría 
la teoría clásica del comercio,

2. o desde el efecto multiplicador del comercio exterior, como sugiere la teoría moderna,

3. o a nivel de la larga lista de los llamados efectos educativos del comercio, frecuentemente discutidos en la literatura". p. (343).

Papanek (1972 y 1913), menciona explícitamente una razón importante de porque la $\mathrm{PMgS}$ será diferente entre los dos sectores. La propensión marginal a ahorrar de los ingresos de el sector exportador será más alta que la $\mathrm{PMgS}$ de otros sectores, porque en el caso de las exportaciones básicas, hay generalmente un elemento importante de renta, y los ingresos están altamente concentrados en este sector.

Con todos estos puntos, es razonable aceptar la especificación de la función de ahorro ex-ante propuesta por Maizels (1968):

$$
S t=C+d(Y t-X t)+e X t
$$

donde $\mathrm{Xt}$ son los ingresos del sector exportador y $(\mathrm{Yt}-\mathrm{Xt}$ ) son los ingresos del sector no exportador. Maizels, sostiene que esta especificación es más apropiada, porque si los ahorro son especificados como en la ecuación (1), en el modelo de las dos brechas, la brecha del ahorro esta sobrecondicionada, puesto que un incremento en las exportaciones solo afectará la brecha comercial, dejando la primera sin afectar.

En la segunda línea de argumentos, en el modelo de dos brechas da una explicación de porque nosotros encontraríamos una fuerte correlación entre exportaciones y ahorro Chenery y Bruno (1962). Mckinnon (1964). Chenery y Strout (1966). Chenery y Eckstein (1970). Este modelo emplea la siguiente identidad derivada de la contabilidad nacional:

$$
(\mathrm{It}-\mathrm{St})=(\mathrm{Mt}-\mathrm{Xt})
$$

El lado izquierdo es la brecha de ahorro, (It - St), la cual muestra el exceso de inversión sobre el ahorro nacional. El lado derecho es la brecha comercial, (Mt - Xt), la cual expresa el exceso de im- 
portaciones sobre las exportaciones. Las entradas extranjeras juegan un doble papel en este contexto. Ellas proveen la fuente necesaria para financiar un nivel de inversión compatible con un objeto de tasa de crecimiento llenando la brecha de ahorro. $Y$ ellas completan con la divisa el papel de las exportaciones en el financiamiento de las importaciones de capital y bienes intermedios necesarias para alcanzar una tasa de crecimiento dado, llenando la brecha comercial. Por consiguiente, mientras ambas brechas sean diferentes, ex-post son iguales. En este contexto la tasa de crecimiento es forzada por cualquiera que sea la brecha más grande. $\mathrm{Si}$ la brecha comercial es la predominante, la brecha de ahorro será ajustada a través del más alto consumo superfluo y el deseo ignorado de ahorro; por consiguiente, las observaciones históricas de ahorro no corresponden a la función de ahorro ex-ante, mientras las observaciones en importaciones corresponden a las estimaciones ex-ante.

Si la brecha de ahorro es la predominante, la brecha comercial será ajustada a partir de un nivel de importaciones más alto que el necesario para alcanzar la tasa de crecimiento objetivo; por tanto, las observaciones históricas de las importaciones no corresponden a la demanda ex-ante de importaciones, mientras las observaciones en ahorro corresponden a las estimaciones ex-ante.

Si la brecha comercial es la predominante, un incremento en las exportaciones operará con escasos recursos, i. e., divisas y si la parte de importaciones en bienes de capital es muy alta, permitirá una realización de niveles más altos de ahorro nacional deseado. Por eso en este caso esperaríamos observar una correlación positiva entre exportaciones y ahorro.

Siguiendo a Maizels (1968), una versión simplificada de el modelo Chenery-Strout puede ser formalizada. Puesto que estamos interesados en el comportamiento del ahorro, nos limitamos a resolver solo la ecuación de ahorro. El nivel objetivo de producción total para cada año, $Y^{*}$, es dado por una tasa de crecimiento objetivo, $\mathrm{r}^{*}$ :

$$
Y^{*} t=Y_{0}\left(1+r^{*}\right)^{t}
$$


El nivel de inversión necesaria para conseguir este nivel objetivo de producción total es dado por la relación incremental capitalrendimiento de capital, \&, como sigue:

$$
\mathrm{I}_{\mathrm{t}}^{*}=\& \mathrm{r}^{*} \mathrm{Y}_{\mathrm{t}}^{*}
$$

La tasa de crecimiento de las exportaciones $\mathrm{E}$, es dada exogenamente de modo que el nivel de exportaciones es:

$$
X_{t}=X_{o}(1+E)^{t}
$$

El nivel deseado de ahorro es dado por la propensión marginal a ahorrar ex-ante:

$$
S_{t}^{*}=S o+S_{1}^{*} Y_{t}^{*}
$$

El nivel mínimo de importaciones para alcanzar el nivel de producción total objetivo es dado por:

$$
M_{t}^{*}=m_{0}+m_{1}^{*} Y^{*}
$$

$Y$ finalmente, cuatro identidades ex-post de contabilidad nacional aproximan este modelo:

$$
\begin{aligned}
Y_{t} & =C_{t}+I_{t}+X_{t}-M_{t} \\
Y_{t} & =C_{t}+S_{t} \\
I_{t} & =S_{t}+F_{t} \\
M_{t} & =X_{t}+F_{t}
\end{aligned}
$$

De estas cuatro identidades, solo tres son independientes, por lo tanto podemos reemplazar las identidades (11) y (12) por:

$$
\text { It }- \text { St }=\text { Mt }-X t
$$

La cual es una identidad ex-post. Ahora, la brecha de ahorro exante es dada por $\left(\mathrm{I}^{*}-\mathrm{S}^{*}\right)$, y la brecha comercial ex-ante por $\left(\mathrm{M}^{*}-\mathrm{X}\right)$. 
Cuando la brecha de ahorro ex-ante es más grande que la brecha comercial ex-ante, la ecuación (7) se vuelve operativa, mientras que la ecuación (8) no lo es. Hasta ahora la observación histórica del ahorro corresponde a la función de ahorro ex-ante, cuando la brecha de ahorro es predominante, y por lo tanto la ecuación apropiada para un estudio empírico sería la ecuación (7).

$\mathrm{Si}$ la brecha comercial ex-ante es más grande que la brecha de ahorro ex-ante la ecuación (8) se vuelve operativa, y la ecuación (7) no. Así, resolvemos el modelo para la ecuación de ahorro cuando la brecha comercial es predominante. Substituyendo (5) y (8) en (13):

$$
S t=-m o+\left(\& r^{*}-m 1\right) Y t+X t
$$

La cual sería la ecuación apropiada para una estimación empírica de la función de ahorro si la brecha de comercio es la predominante.

$\mathrm{Si}$ la verdadera función ahorro ex-ante es mejor justificada por una distinción entre la propensión marginal a ahorrar entre el sector exportador y el sector no exportador, entonces podemos substituir la ecuación (7) por:

$$
S_{t} \neq S_{0}+S_{1}^{*}\left(Y_{t}-X_{t}\right)+S_{2}^{*} X_{t}
$$

Con esta modificación, si la brecha comercial es operativa, la ecuación apropiada para explicar el comportamiento del ahorro es todavía la ecuación (14). Si la brecha de ahorro es la predominante, la ecuación apropiada sería la (15).

En el caso extremo, cuando asumimos que todo el ahorro proviene de los ingresos generados por el sector exportador, que es $S 1=0$, la ecuación adecuada para el régimen obligado de comercio es todavía la ecuación (14). Mientras la ecuación adecuada para el régimen obligado de comercio es:

$$
S_{t}=S_{0}+S_{2}^{*} X
$$

Además, para propósitos empíricos podemos ver que si los ahorros deseados son descritos por la ecuación (16, y asumimos que 
durante el período de estimación los dos regímenes no alternaron, y uno solo es el predominante, la ecuación lineal:

$$
S_{1}=\alpha_{0}+\alpha_{1} Y_{t}+\alpha_{2} X_{1}+V_{t}
$$

provee una forma para determinar cual brecha fue la preponderante. Si $\alpha$ es igual a cero, entonces la brecha de ahorro es preponderante y $\alpha_{2}$ da la propensión marginal a ahorrar ex-ante de los ingresos de el sector exportador. Por otra parte si a es mayor que cero y $\alpha_{2}$ es igual a 1 la brecha comercial fue la preponderante.

Mamalakis (1970), en un estilo muy similar al modelo de dos brechas explica la relación entre las exportaciones y el ahorro, a través del papel de las exportaciones como "quiasi-bienes de capital". En una economía donde el sector de bienes de capital esta casi ausente, el papel de las exportaciones es muy importante, puesto que prevee las divisas para financiar las importaciones de bienes de capital. En ese sentido, el sector exportador es considerado como un sector de bienes de quasi capital, el cual provee a la economía con la posibilidad de acumulación capital. Tomando en cuenta la ecuación derivada de la contabilidad nacional:

$$
S_{t}=I_{t}-\left(M_{t}-X t\right)
$$

Un aumento en las exportaciones permitirá importar bienes de capital e incrementará el nivel de inversión, dejando inalterada la brecha de comercio, e implicando niveles más altos de ahorro nacional en esta identidad.

En ambos casos, el modelo de dos brechas y Mamalakis (1970), la correlación entre exportaciones y ahorro es apoyado solo si la brecha de comercio es obligatoria, y en ese sentido lo que es observado es el ahorro ex-post.

Finalmente, en una tercera clase de razonamiento, si los ingresos generados por las exportaciones son inestables, lo cual es generalmente el caso de los países menos desarrollados, generaría un tipo de efecto de ingresos permanentes para ahorro. Puesto que los intentos individuales por un consumo suave, en buenos años, donde hay un alza en las entradas de exportación, exportadores 
incrementarán sus ahorros para los años malos. En años donde las entrañas de exportación caen, los ahorros caerán. En ese sentido, si la brecha de ahorro es forzada, de modo que el ahorro ex-ante corresponde al ahorro ex-post, habrá un correlación entre exportaciones y ahorro como Chenery y Eckstein expusieron: "Esto sería verdad si las amplias fluctuaciones en los ingresos de exportación caen principalmente en ganancias, o si ellos crean un efecto general de ingreso permanente. . ." (Chenery y Eckstein, 1970).

Resumiendo en una economía de exportaciones primarias, con un alto porcentaje de exportaciones en el producto nacional bruto, una alta dependencia en importaciones de capital y bienes intermedios y con una alta concentración de ingresos en el sector exportador, hay diversas razones técnicas para sostener una alta correlación entre exportaciones y ahorro. Esta justificación puede ser encontrada en una sencilla función de ahorro de la Teoría Keynesiana como en Maizels (1968), Lee (1971) y Papanek (1972-1973), o en un modelo más completo como en Mamalakis (1970) o como en el modelo de dos brechas propuesto por Hollis B. Chenery y asociados. En la estructura de dos brechas, si las exportaciones no son incluidas en la función de ahorro ex-ante, la correlación entre exportaciones y ahorro se mantendrá solo si la brecha comercial es forzada. Pero si las exportaciones son incluidas en la función de ahorro ex-ante, como en Mamalakis o si ay alguna clase de efecto de ingreso permanente en el sector exportador, la correlación entre exportaciones y ahorro se mantendrá en las dos situaciones, pero será mayor si la brecha de comercio es la predominante.

\section{Evidencia Empírica de la Relación Ahorro-Exportaciones}

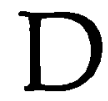
iversos documentos han tratado de verificar la hipótesis que cambios en el ahorro son explicados por los movimientos en las ganancias de las exportaciones. Estos pueden ser divididos en dos grupos. Aquellos que explícitamente tratan de probar la hipótesis Maizels-Lee, y aquellos que incorporan las exportaciones en sus ecuaciones bajo diferentes contextos.

Con respecto al primer grupo, un resumen de los resultados acerca de la significancia de la propensión marginal a ahorrar. PMgS de las entradas nacionales, PMgSy, la PMgS de los ingresos del sector exportador PMgSx, y la PMgS de las entradas de el sector 
no exportador PMgSnx, esta presentado en la tabla 1.

Tabla 1

Resultados para un nivel de significación del $5 \%$ (o menos) de la hipótesis de Maizels-Lee

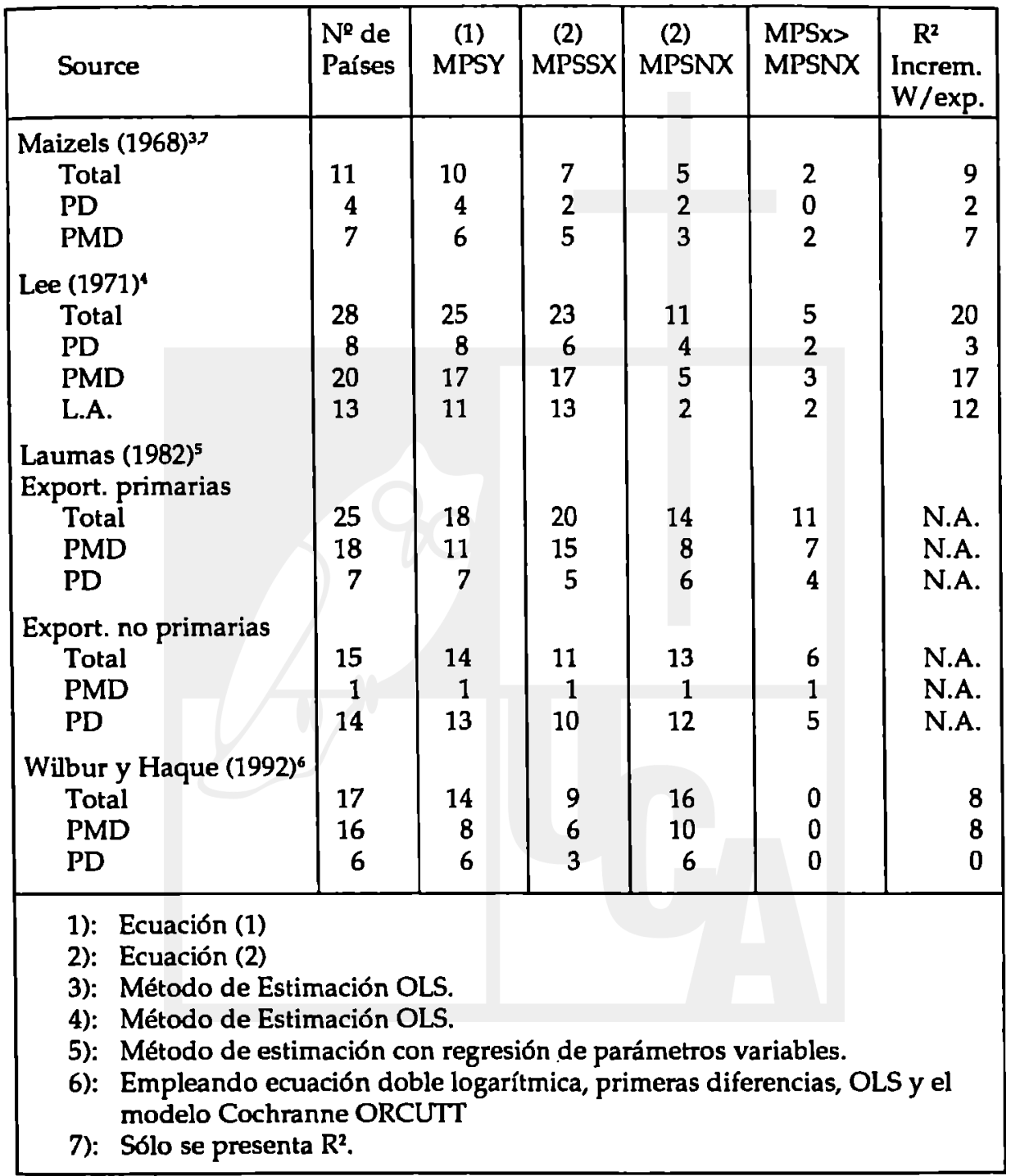


Esta tabla presenta el número de casos de países que tienen una tasa significativa de PMgS. Por ejemplo, la primera fila nos dice que Maizels (1968) estimo ecuaciones para once países. De estos 11 países, 10 tienen una PMgSy significativa usando la sencilla función Keynesiana que es la ecuación (1). Siete tienen una significativa PMgSx, y 5 una significativa PmgSnx, usando la ecuación (2). La PmgSx fue más significativa que la $\mathrm{PMgSnx}$ en dos casos y que el $R^{2}$ mejoré en nueve casos cuando las entradas de exportación fueron incorporadas a la función de ahorro.

Tomando en cuenta esta tabla, si la comparación esta hecha basada sobre el $R^{2}$, o el $R^{2}$ ajustado en la última columna, es clave que para la gran mayoría de los países menos desarrollados. L.D.C.S., la bondad de ajuste mejora cuando el sector exportador es adicionado explícitamente a la función de ahorro; mientras para los países desarrollados, D.C.S., este no es el caso. Es también evidente que en L.D.C.S., la significación estadística es encontrada en más casos para la PMgSx, que para la PMgSnx. Cinco casos contra tres, en Maizels (1968); 17 contra 5 en Lee (1971); 16 contra 9 en Laumas (1982). Willbur y Hauge (1992), es una excepción con 6 contra 10 . Sin embargo, la especificación de su modelo es diferente que la de los otros tres documentos. Esta tendencia no es encontrada en el caso de los D.C.S. Finalmente, no sirve de mucho el hecho de que la hipótesis de Maizels-Lee, i.e. PMgSx PMgSnx, es verificada solo en pocos casos. Dos casos en Maizels (1968), cinco casos en Lee (1971), once casos en Laumas (1982), y cero casos en Willbur y Haque (1992). Por lo tanto podemos concluir que a pesar del hecho que la hipótesis Maizels-Lee no puede ser verificada en una gran mayoría de los casos, la introducción del sector exportador, como una fuente separada de ingresos, lleva a un conciben' mejoramiento en la estimación de la función ahorro en L.D.C.S.

En el otro grupo, hay solo un documento que incorpora las exportaciones en la función de ahorro. Chenery y exkstein (1970). Como ya fue mencionado, la introducción de exportaciones es consistente con el modelo de dos brechas si la brecha de comercio es forzada o si el ingreso de exportación es generado para tener un fuerte efecto en la función de ahorro ex-ante. También incorporan el ahorro extranjero como un determinante del ahorro nacional; puesto que puede ser considerado como un "sustituto perfecto" 
del ahorro nacional? Por eso Chenery y Eckstein estimaron la siguiente ecuación para 16 países latinoamericanos.

$$
S_{t}=a+b Y_{t}+c X_{t} / Y_{t}+d F_{t}
$$

La razón para introducir las exportaciones como una relación del producto nacional bruto, fue solo para evitar problemas de multicolinealidad, con el mismo producto nacional bruto. $\mathrm{Y}$ también estimaron la función simple de ahorro keynesiano en la ecuación (1). Comparando ambas ecuaciones, usando el criterio $R^{2}$, se encontró que en 14 casos fue más alto para la ecuación (19). En esta ecuación, el coeficiente $c$, solo fue significativo y positivo en 7 casos. En una nota a pie de página, Chenery y Eckstein escribieron en 1970:

"Tratamos de estimar ecuaciones en las cuales el ahorro era considerado una función del ingreso de no exportación, del ingreso de exportación y del capital extranjero. Menos digno de confianza, indicaron para cada pais una propensión marginal a ahorrar del ingreso de exportación más grande que del ingreso de no exportación. El promedio para el primero fue 0.61. Para el último solo 0.057. En dos casos México y Argentina, la propensión a ahorrar del ingreso de exportación fue mayor que 1 , y en cuatro casos mayor que 0.6 resultados que son más fácilmente interpretados como causa de una brecha de comercio obligatoria". (p. 976)

Como el primer grupo, estos resultados sugieren que las exportaciones juegan un papel substancial en la explicación del comportamiento del ahorro.

\section{Función de ahorro estimada para El Salvador}

Varios documentos han estimado diferentes funciones de aho Iro, para diferentes periodos, para El Salvador. La función Keynesiana simple fue estimada en Chenery y Eckstein (1970) y en Mikesell y Zinser (1973).

La ecuación estimada por Chenery y Ecktein (1970) empleando OLS; produjo los siguientes resultados; 


$$
\begin{aligned}
& S=107.84-0.07 Y \\
& (3.39)^{* *} \quad(-1.25) \\
& R^{2}=0.0
\end{aligned}
$$

El resultado para la propensión marginal a ahorrar es insignificante y con el signo equivocado. La ecuación estimada por Mikesell y Zinser (1973) usando OLS es:

$$
\begin{aligned}
\mathrm{S}= & \begin{array}{l}
42.72 \\
(1.149)
\end{array}+0.087 \mathrm{Y} \\
(4.282)^{* *} & \\
\mathrm{R}^{-2}= & 0.634
\end{aligned}
$$

La PmgSy fue significativa y menor que uno. Aplicando OLS Mikesell y Zinser también estimaron la función ahorro usando el logaritmo de las variables:

$$
\begin{aligned}
& \operatorname{LnS}=-1.129+0.085 \operatorname{Ln} Y \\
& (-0.811) \quad(4.597)^{* *} \\
& \mathrm{R}^{-2}=0.668
\end{aligned}
$$

La elasticidad ingreso del ahorro fue significativa y menor que uno lo cual significa que la propensión marginal a ahorrar es menor que la propensión media a ahorrar.

Otros documentos han introducido las exportaciones en la función de ahorro. Chenery y eckstein incorporaron las exportaciones en una segunda ecuación:

$$
\begin{aligned}
S= & -118.2+0.08 Y+173.0 X / Y-0.95 F \\
& (-1.1442)(3.12)^{* *}(1.39)(9.96)^{* *}
\end{aligned}
$$

Guiado por una estimación más confiable, donde el coeficiente asignado al producto nacional bruto, $\mathrm{y}$ al ahorro extranjero produjo valores significativos; mas un $R^{2}$ mayor comparado con un $R^{2}$ cero. Aunque el coeficiente no es significativo, en este caso la introducción 
de las exportaciones como una variable explicatoria mejora el $R^{2}$.

Landau (1971), también incorporo el ahorro extranjero a la función Keynesiana simple, sin embargo, solo presento una de las estimaciones de la OLS:

$$
S=a+b Y-\frac{0.0603 F}{(5.482)^{* *}}
$$

Obteniendo un resultado significativo para $\mathrm{Ft}$; como el coeficiente para esta variable en el modelo Chenery.

No obstante, este resultado acerca del coeficiente en Ft debería ser interpretado con precaución. Papanek (1972-1973) sostiene que hay muchos factores que afectan la relación entre Ft y St. Primero aquellos que están en favor de una relación negativa entre estas dos variables sostienen que los ahorros están íntimamente relacionados con la política gubernamental, así que los esfuerzos fiscales de ahorro gubernamental serán menores si mas recursos externos entran en la economía; y que si los ahorros son función de las oportunidades de inversión, y si algunas de estas oportunidades son cubiertas por capital extranjero, entonces la entrada de capital extranjero como inversión directa expulsará el ahorro nacional.

Segundo, Papanek sostiene que puede haber otro conjunto de funciones de ahorro que pueden producir un incremento en el ahoro y la inversión como resultado de entradas de capital extranjero: (a) si la inversión es función de la divisa disponible para la importación de bienes de capital e intermedios para mantener la economía trabajando a plena capacidad entonces las entradas de capital extranjero permitirán un incremento de la inversión, así como ahorro e ingresos; (b) Si los ahorros son una función del nivel y la tasa de crecimiento del ingreso en sectores específicos, tales como industriales y exportadores, las entradas de capital extranjero pueden incrementar los ahorros elevando los ingresos de estos grupos, aun cuando el nivel medio de ingresos es bajo; (c) Si los ahorros son una función de los ingresos y el gobierno no tiene un mecanismo efectivo para reducir los ahorros en orden a balancear el incremento de inversiones financiadas directamente por entradas de capital extranjero el efecto neto en la inversión dependerá de la 
proporción de entradas de capital extranjero que son usadas para la misma.

Tercero, la relación negativa entre St y Ft no es siempre explicada por una relación causal, puesto que ambas pueden estar relacionadas por una tercera variable. Por ejemplo, en un país pobre de acuerdo a la tesis tradicional los ahorros serán bajos, pero debido a la urgente necesidad por ayuda es altamente probable que el país vaya a recibir más ayuda extranjera (esto es especialmente relevante para un análisis a campo traviesa); también, si un país esta en una situación de guerra civil o de graves disturbios políticos, los ahorros caerán, pero la ayuda extranjera puede aumentar si el país es estratégicamente importante para los paises donantes; un drástica caída en los términos de intercambio o un desastre natural, puede tener el mismo efecto de una caída en el ahorro, acompañada por un incremento en la ayuda extranjera.

Cuarto, muchos de los estudios que encontraron una relación negativa entre St y Ft sostienen que todas las entradas de capital extranjero están orientadas a la inversión, pero esto no se aproxima a la realidad, puesto que una buena parte de los recursos extranjeros son usados para consumo de bienes e insumos necesarios para la producción. En ese sentido cuando estimamos los ahorros por la regla $S_{t}=I_{t}-F_{t}$ no es correcto reducir el ahorro nacional por la cantidad de entradas de capital extranjero que son recibidas como ayuda con propósitos de consumo.

Finalmente, la introducción de $F_{t}$ en el modelo puede conducir a un problema de baja identificación como fue notado por Leff y Sato (1988). Especialmente, porque $I_{1}$ y Ft pueden ser relacionadas por terceras variables, y porque el ajuste de las tres variables implicadas en la identidad $\mathrm{It}+\mathrm{St}+\mathrm{Ft}$, son parte de un proceso simultáneo. Por lo tanto la escasa identificación es relacionada con problemas de simultáneidad y restricciones más específicas en los parámetros.

Wilbur y Haque (1992) es el único documento que ha probado la hipótesis Maizels-Lee para El Salvador, para el período 19601987, pero en lugar de usar los valores absolutos de las variables, uso la primera derivada de el logaritmo de las variables, y aplico el procedimiento Cochrane-Orcutt para corregir la autocorrelación. 
Cuando usaron solo el GDP como una variable explicatoria, el coeficiente fue significativo y el $R^{2}$ ajustado fue alto:

$$
\begin{aligned}
\operatorname{Ln} S= & -10.1018+1.657 \operatorname{Ln} Y \\
& (12.0495) \quad(2.085)^{*} \\
R^{-2}= & 0.962
\end{aligned}
$$

Cuando usaron como variables explicatorias los ingresos de el sector no exportados, y los ingresos del sector exportador, solo el coeficiente atribuido para el último fue significativo y hubo una mejora en el $R^{2}$ ajustado:

$$
\begin{aligned}
\operatorname{LnS} S_{t}= & -3.2556+0.3104 \operatorname{Ln}\left(Y_{t}-X_{t}\right)+0.9541 \operatorname{Ln} X_{t} \\
& (1.33124)(1.0316) \\
R^{-2}= & 0.974
\end{aligned}
$$

Por lo tanto, insinuando que en El Salvador, los ingresos provenientes del sector exportador deberían ser incorporados explícitamente en la función ahorro; esta debería ser corroborada mediante algunas pruebas de error de interpretación.

Leff y Sato (1988), no usaron la exportación como una variable explicatoria directa, aunque fue incluida en su conjunto instrumental de variables. Su ecuación es:

$$
S=a+b \Delta Y_{t}+c P_{t}^{e}+d S_{t-1}
$$

Donde pe significa las expectativas de inflación, y es la primera derivada técnica. La ecuación (27), fue derivada de un modelo de acumulación de capital. Vieron al ahorro como un mecanismo empleado por los individuos para ajustar sus tenencias de capital a un nivel de deseado de riquezas:

$$
S_{t}=B\left(W^{*}-(1-\&) W t-1\right) \quad 0<B \leq 1
$$

Donde $W_{t}^{*}$ es el nivel deseado de riqueza en el periodo $t, W_{t-1}$ 
es la riqueza en el período previo, \& es la tasa de depreciación de la riqueza, y es el coeficiente de ajuste. El nivel deseado de riqueza es dado por la relación capital-ingreso objetivo $W_{t}^{*}=W_{t}^{*} / Y_{t}^{*}$. Esta relación después de algo de algebra, justifica la incorporación de $Y_{t}$ y $S_{t-1}$. Con respecto a la inflación esperada, encontraron cuatro razones para añadirla como una variable explicatoria del ahorro: (a) Un efecto de balance verdadero debido a la necesidad de la existencia del índice de inflación del capital, la cual induce a los individuos a ahorrar más para mantener su liquidez en una situación de activos reales: (b) mayores expectativas de inflación implican expectativas de mayor incertidumbre, generando un cauteloso incremento en el ahorro; (c) Puesto que la tasa de interés y el tipo de cambio pueden ajustarse lentamente a mayores tasas de inflación, los individuos pueden incrementar el consumo de importaciones o de bienes domésticos durables y (d) el efecto negativo de la inflación esperada en las tasas reales de interés puede que reflejado en menores ahorros. Mientras ( $a$ ) y (b) tienen un efecto positivo en el ahorro, (c) y (d) tienen un efecto negativo, por lo tanto el signo del coeficiente de la tasa de inflación esperada en el función de ahorro es dejado a la evidencia empírica.

Usando este modelo para estimar la función de ahorro ex-ante para El Salvador, para el período 1955-1983, aplicaron tres escenarios menos rígidos y el procedimiento Hatanaka para corregir la autocorrelación..$^{5}$ La ecuación estimada es:

$$
\mathrm{S}=\underset{(2.12)^{*}}{2.27 \mathrm{E}+01+}+\frac{0.566 \Delta \mathrm{Y}+3.36)^{* *}}{(5.42)^{* *}}+\frac{5.376 \mathrm{E}+02 \mathrm{pe}+}{(5.57)^{* *}}
$$

Los resultados sugieren que en El Salvador, un comportamiento dinámico para el ahorro ex-ante es posible y que la inflación esperada tiene un efecto significativo en el ahorro.

Finalmente, los modelos presentados por Leff y Santo (1988), y Wildur y Haques (1992), podrían estar mal interpretados puesto que abarcaron un período que cruzaba la década de los ochenta. $Y$ en estos años el golpe de la guerra fue reflejado en un notable cambio descendente en todas las variables involucradas en sus modelos excepto la inflación esperada. Sin embargo, esto puede ser 
corregido añadiendo una variable aleatoria cubriendo los periodos de inestabilidad política y guerra.

\section{Capitulo II}

La importancia de los ingresos de exportación en el comportamiento del ahorro en El Salvador

F ste capítulo trata de demostrar en una forma indirecta, que el Eahorro en El Salvador proviene esencialmente de las ganancias del sector exportador. En orden a hacer esto, primero intento determinar cual brecha es forzada para poder diferenciar, para cada año, si el ahorro observado corresponde a la función de ahorro ex-ante o no. En una segunda parte, una serie de pruebas econométricas son mostradas para verificar que la principal fuente de ahorro es el ingreso proveniente del sector exportador. En el resto de esta sección, los datos cubre el período 1958-1991, y son tomados de las "Estadísticas Financieras Internacionales" del FMI y de la revista mensual del Banco Central de El Salvador (Ver apéndice A). A menos que se señale de otro modo, el método de estimación es el OLS.

El régimen dominante en el modelo de dos brechas

Duesto que en la estimación de la función ahorro es necesario ante o ahorro ex-post, es importante saber cual brecha es forzada. Diversos documentos han tratado de estimar cual brecha es forzada. Esta sección hace esa prueba con dos planteamientos diferentes, uno propuesto por Landau (1971), y el otro por Chenery u Eckstein (1970).

Sin embargo, estos dos planteamientos no son consistentes con el modelo de dos brechas, puesto que básicamente ignoran que los dos regímenes pueden alternar a lo largo del período de estimación. El mejor intento para derivar el régimen dominante para cada período, es dado por un método de estimación para modelos en desequilibrio sugerido por Maddala y Nelson (1971), y aplicado al modelo de dos brechas por Gersonitz (1982). Aplique esta prueba, pero los resultados proporcionaron valores extremos de modo que consideraré las otras dos pruebas solo como una estimación burda. 
Landau presenta el modelo de dos brechas con dos funciones ex-post una para el ahorro y la otra para las importaciones:

$$
\begin{aligned}
& S_{t}=a_{1}+b_{1} Y_{t}+\alpha_{1} F_{t} \\
& M_{t}=a_{2}+b_{2} Y_{t}+\alpha_{2} F_{t}
\end{aligned}
$$

Según el, si la brecha de ahorro es forzada durante todo el periodo estimado entonces $\alpha_{1}$ es igual a cero, puesto que las observaciones históricas del ahorro corresponden a la función de ahorro ex-ante que es la ecuación (7). $\alpha_{2}$ es positivo puesto que las entradas adicionales de capital extranjero permitirán importar por encima del nivel requerido. Si la brecha de comercio es forzada, entonces $\alpha_{1}$ es negativa puesto que las entradas adicionales de capital extranjero serán un substituto para el ahorro nacional; y $\alpha_{2}$ es cero puesto que estoy observando el nivel de importaciones dado por la ecuación (8). Si durante el período de estimación los dos regímenes estuvieron alternando este procedimiento se vuelve más difícil, y las estimaciones de $\alpha_{1}$ y $\alpha_{2}$ pueden ser simultáneamente diferentes de cero.

Tomando en cuenta la variable aleatoria por la guerra, aplique este procedimiento para el caso de El Salvador durante el período 1958-1991. Puesto que encontré un problema de autocorrelación en el término de perturbación, utilicé el comando AR (1) en MicroTSP 7.0, por lo que la estimación actual corresponde al período 1959-1991. Las ecuaciones (30) y (31) proporcionan los siguientes resultados:

$$
\begin{aligned}
\mathrm{S}_{\mathrm{THX}}= & -297.4+0.293 \mathrm{Y}_{\mathrm{t}}-0.855 \mathrm{~F}_{\mathrm{t}}-212.8 \text { Guerra } \\
& (3.12)^{* *} \quad(7.57)^{* *}(9.29)^{* *}(5.06)^{* *} \\
\mathrm{R}_{2}^{2}= & 0.882 \text {, } \text { D.W. }=2.20
\end{aligned}
$$

Nuestros valores de interés $\alpha_{1}=0.865$ y $\alpha_{2}=0.403$, son ambos significativamente diferentes de cero en un nivel de significación de $1 \%$ sugiriendo que El Salvador se enfrenta con regímenes alternativos durante este período, y por lo tanto los otros co- 
eficientes en ambas ecuaciones no representan los de las funciones ex-ante.

Tomando en cuenta esta evidencia, trate de determinar cual brecha fue siguiendo a Chenery y Eckstein (1970). Para estimar la brecha de ahorro, primero estimaron la siguiente ecuación, tomando $\mathrm{Ft}$ como una variable exógena:

$$
S_{t}=a+b Y_{t}+c X_{t} / Y_{t}+d F_{t}
$$

Entonces, asumiendo que $a, b, y$ c son coeficientes de ahorro deseado y omitiendo F. pronosticaron el ahorro ex-ante como sigue:

$$
S_{t}=S_{t-1}+b \Delta Y_{t}+c \Delta X_{t} / Y_{t}
$$

Ya que estos tratando con datos observados, en lugar de estimar la inversión necesaria para alcanzar la tasa observada de crecimiento del PNB, puedo tomar la inversión como dada, y la brecha de ahorro será dada por la diferencia entre el ahorro potencial o ex-ante y la inversión.

Para estimar la brecha de comercio, puedo suponer las exportaciones como variables exógena. Basado en el supuesto que hay una alta dependencia de las importaciones de bienes de capital y la insuficiencia de divisas representada por su disponibilidad, la siguiente ecuación es para las importaciones requeridas:

$$
M_{t}=f+g C_{t}+h I_{t}+i X_{t} / Y_{t}+j R_{t} / Y_{t}
$$

Donde Rt es el valor real de las reservas internacionales en moneda nacional. La presencia de Xt y Rt es importante en el modelo Chenery-Ecktein, ya que ellos observaron que cuando la divisa es escasa el gobierno trata de racionar su uso. Forzando a los consumidores nacionales a substituir bienes domésticos por importados. En ese caso, cuando las relaciones de exportaciones sobre ingresos y reservas sobre ingresos son pequeñas, ya que el gobierno es selectivo en el uso de la divisa, la propensión marginal a importar de consumo e inversiön, $\mathrm{g}$ y $\mathrm{h}$ respectivamente son altas. $\mathrm{Si}$ las últimas dos variables son omitidas, $\mathrm{g}$ y $\mathrm{h}$ son subestimadas. Entonces pue- 
do estimar la brecha de comercio substrayendo las exportaciones de las importaciones necesarias, las cuales son obtenidas usando la primera derivada de la ecuación (36):

$$
M_{t}=M_{t-1}+g \Delta C_{t}+h \Delta I_{t}+i \Delta X_{t} / Y_{t}+j \Delta R_{t} / Y_{t}
$$

Los resultados para las ecuaciones (36) y (37) son:

$$
\begin{aligned}
\mathrm{S}_{1}= & 392.4+0.20 \mathrm{Y}_{\mathrm{t}}+1111.2 \mathrm{X}_{\mathrm{t}} \mathrm{Y}_{\mathrm{t}}-0.56 \mathrm{~F}_{\mathrm{t}}-160.3 \text { Guerra } \\
& (7.81)^{* *}(6.79)^{* *}(3.94)^{* *} \quad(4.51)^{* *}(5.54)^{* *} \\
\mathrm{R}= & 0.918 \text {. D.W. }=1.42
\end{aligned}
$$

$(7.81)^{* *}(6.79)^{* *} \quad(3.94)^{* *} \quad(4.51)^{* *}(5.54)^{* *}$

$$
\begin{aligned}
M_{1}= & -499.7+0.21 C_{1}+0.72 J_{1} / Y_{1}+1813 X_{1} / Y_{1}+186.9 R_{1} / Y_{1}+49.12 G_{1} \\
& (7.77)^{* *}(5.05)^{* *}(6.86)^{* *}(10.5)^{* *}(0.29)^{* *} \quad(1.21)^{* *}
\end{aligned}
$$

$\mathrm{R}^{2}=0.918$, D.W. $=1.42$

Ahora, hago uso de los coeficientes de ambas ecuaciones para estimar ambas brechas. Con la ecuación (38) y tomando las inversiones como dadas, tengo una estimación de brecha de ahorro GS = $\left(I_{t}-S_{t}^{*}\right)$, y con la ecuación (39) y tomando las exportaciones como dadas, tengo una estimación la brecha de comercio $G T=\left(M_{t}^{*}-X_{t}\right)$. Las dos brechas estimadas y la diferencia entre ellas son representadas en la tabla 2. Como se puede ver la brecha de ahorro fue dominante en 21 años de 33 , esto es en $63.6 \%$ de los años. Además en la figura 2, presento el desempeño de las exportaciones y las importaciones tan bien como el balance comercial. Como se puede ver durante los sesentas el déficit comercial fue relativamente pequeño comparado con los ochenta y las exportaciones y las importaciones estuvieron moviéndose a la par, lo cual puede ser considerado como una señal de un régimen en el cual la brecha comercial fue forzada. Esto es consistente con los resultados en la tabla 2 , donde la mayoría de los años con régimen de brecha comercial están en los sesenta. Para 1978 y 1980 la preponderancia de la brecha comercial puede ser explicada por la caída de las exportaciones después de dos puntos máximos de 1977 y 1979. Durante 
Estimación de dos brechas 1959-1991

\begin{tabular}{|c|c|c|c|}
\hline Años & GS & GT & $(G T-G S)$ \\
\hline $\begin{array}{l}1959 \\
1960 \\
1961 \\
1962 \\
1963 \\
1964 \\
1965 \\
1966 \\
1967 \\
1968 \\
1969 \\
1970 \\
1971 \\
1972 \\
1973 \\
1974 \\
1975 \\
1976 \\
1977 \\
1978 \\
1979 \\
1980 \\
1981 \\
1982 \\
1983 \\
1984 \\
1985 \\
1986 \\
1987 \\
1988 \\
1989 \\
1990 \\
1991 \\
\end{array}$ & $\begin{array}{r}-48.652 \\
93.822 \\
6.318 \\
-58.960 \\
-11.792 \\
73.039 \\
21.094 \\
92.492 \\
45.479 \\
-5.563 \\
102.623 \\
84.359 \\
47.270 \\
-35.318 \\
82.174 \\
194.921 \\
253.103 \\
81.817 \\
134.754 \\
103.546 \\
114.894 \\
-46.663 \\
183.803 \\
307.056 \\
184.256 \\
273.959 \\
233.888 \\
342.485 \\
227.952 \\
330.351 \\
366.965 \\
233.614 \\
493.688\end{array}$ & $\begin{array}{r}-24.474 \\
37.576 \\
47.021 \\
1.522 \\
13.908 \\
84.853 \\
50.051 \\
84.834 \\
75.992 \\
25.757 \\
74.708 \\
59.357 \\
45.062 \\
-12.718 \\
43.471 \\
162.921 \\
218.195 \\
55.077 \\
45.941 \\
203.902 \\
76.652 \\
-19.623 \\
141.512 \\
224.647 \\
135.997 \\
190.991 \\
183.985 \\
215.249 \\
209.462 \\
265.377 \\
312.922 \\
266.821 \\
437.843\end{array}$ & $\begin{array}{r}24.178 \\
-56.246 \\
40.703 \\
60.481 \\
25.699 \\
11.814 \\
28.957 \\
-7.658 \\
30.514 \\
31.320 \\
-27.915 \\
-25.002 \\
-2.208 \\
22.600 \\
-38.703 \\
32.000 \\
-34.908 \\
-26.740 \\
-88.813 \\
100.356 \\
-38.242 \\
27.040 \\
-42.292 \\
-82.409 \\
-48.259 \\
-82.968 \\
-49.904 \\
-127.236 \\
-18.490 \\
-64.974 \\
-54.043 \\
33.207 \\
-55.846\end{array}$ \\
\hline
\end{tabular}

los ochenta, debido a las condiciones de guerra y la entrada masiva de ayuda extranjera la brecha comercial es muy grande y no se puede encontrar una relación estrecha entre exportaciones, importaciones y nivel de ingreso que es consistente con la información proporcionada en la tabla 2, en la cual la brecha de ahorro fue dominante en toda la década de los ochentas, con la excepción de 1980 y 1990 . Para 1990 la brecha comercial fue predominante, talvez 
Fig. 2: Importación y exportación 1958-1991

(Miles de colones)

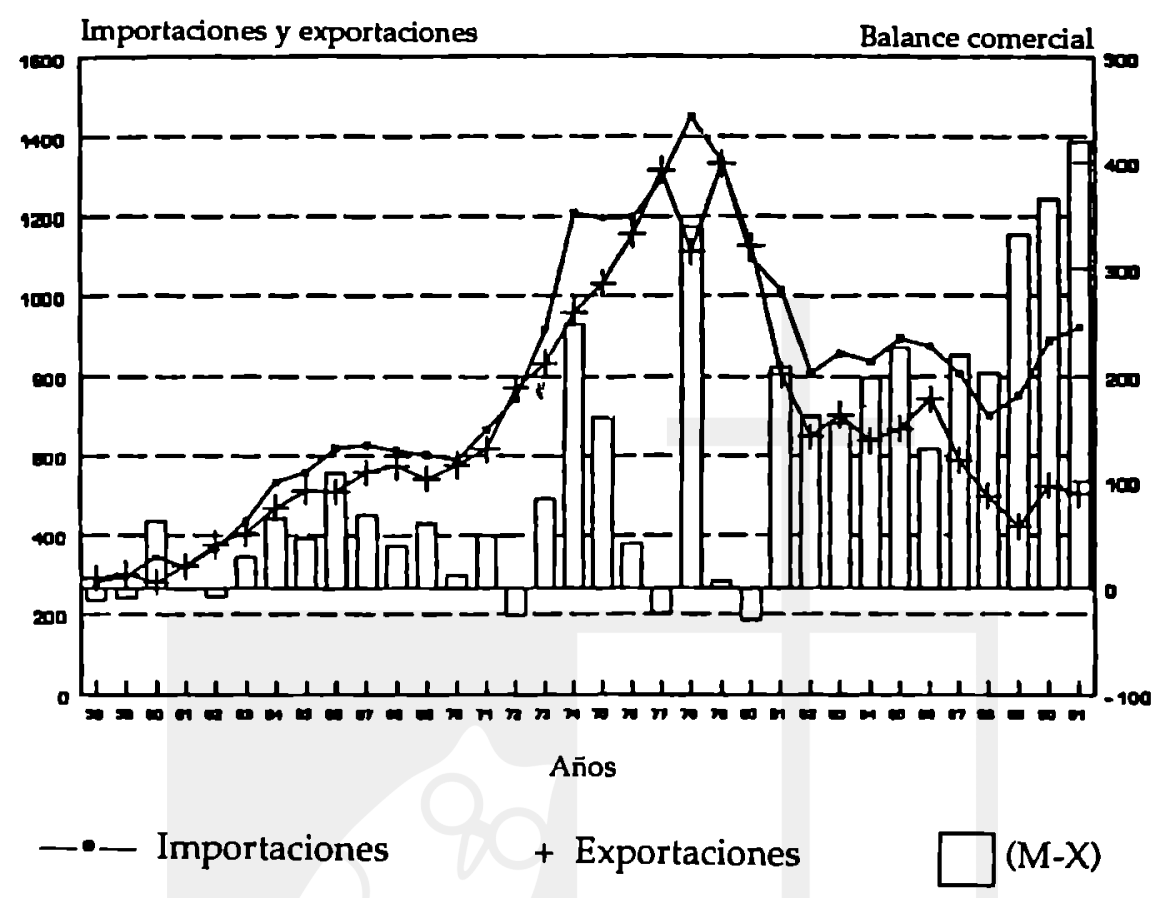

porque hubo un incremento en el nivel de exportaciones, así como en importaciones, indicando una recuperación en la economía por el incremento en las exportaciones.

A pesar de estos resultados, puesto que la brecha de ahorro fue dominante en 21 años de 33 esto es en $63.6 \%$, en la siguiente sección, por simplificación asumiré que estoy estimando la función de ahorro ex-ante. Sin embargo, anotaría que esta será solo una aproximación tosca.

Pruebas estadísticas que favorecen a las exportaciones en contra del PNB como variable explicatoria para el ahorro.

esde el punto de vista econométrico hay diversas maneras de
evaluación para la especificación de un modelo. Aquí, se han
escogido un conjunto de restricciones para verificar la importancia
de las exportaciones en el comportamiento del ahorro en El Salvador. 
En primer lugar, aplique una prueba J, que es una prueba de error de interpretación para verificar cual conjunto de regresiones es el más apropiado para explicar determinada variable. Para ilustrarlo puedo utilizar la teoría Keynesiana simple. En la literatura, el ahorro usualmente se representa como una función lineal del PNB. En nuestro contexto, una hipótesis alternativa, es que el ahorro es una función del ingreso de exportación $X$. En ambas ecuaciones se introduce una variable aleatoria para representar los años de crisis política y guerra: un número es asignado a cada año de 1979 a 1991, y un cero a todos los demás. Tenemos:

$$
\begin{aligned}
& \text { Ho: } S_{t}=a+\text { bPNB }_{t}+\text { cGuerra }_{t}+U_{t} \\
& \text { Hl: } S_{t}=d+\text { eX }+ \text { fGuerra }_{t}+V_{t}
\end{aligned}
$$

Dado que la prueba J es una prueba de error de especificación, se crea una ecuación artificial asumiendo que Ho es cierta:

$$
S_{t}=a+b P N B_{t}+c \text { Guerra }+\alpha_{1}\left(d+e X_{t}+\text { fGuerra }_{t}\right)+W_{t}
$$

Luego se realiza una prueba t simple con $\alpha_{1}=0$. El término en paréntesis es el valor esperado del ahorro proveniente de $\mathrm{Hl}$.

Si $\alpha_{1}=0$, la hipótesis nula puede ser aceptada, y si a1 $\neq 0$, la Ho es rechazada.

Una diferencia de esta prueba es que se revierte el papel de la hipótesis, esto es:

$$
\begin{aligned}
& H 3: S_{t}=d+e X_{t}+\text { fGuerra }_{t}+V_{t} \\
& H 4: S_{t}=a+\text { bPNB }_{t}+c \text { Guerra }_{t}+U_{t}
\end{aligned}
$$

Debo mencionar que el coeficiente a probar en la ecuación establecida se relaciona con las ecuaciones (42), $\alpha 0$. Entonces, surgen 4 posibilidades: aceptar cualquiera de las funciones, rechazar ambas o aceptarlas.

Durante la estimación la ecuación en Ho. Presentó problemas de 
auto correlación en el término de error. Esto puede haber sucedido debido a la omisión de una variable relevante, v.gr. Xt. Dado que quiero comparar esto con $\mathrm{hl}$, necesite un método de estimación que me permitiera estimar los parámetros para todo el rango de observaciones. Por ello, los utilice el método Prais-Winsten que es un método de dos pasos (FGLS completo: feasible generalized least squares). (Greene (1993). p. 431). Porque la suposición inicial es que Ho, es cierta, el procedimiento es adecuado, a pesar de la posibilidad de un error de interpretación. La interpretación usual de $R$ no es valida para esta ecuación. Las ecuaciones estimadas son: Las dos primeras ecuaciones son las dos hipótesis alternativas, $\mathrm{Ho} \mathrm{y} \mathrm{Hl}$ mientras que las otras dos son las ecuaciones artificiales.

$$
\begin{aligned}
& S_{t}=-148.49=0.19 Y_{t}-265.1 \text { Guerra }_{t} \\
& \text { (1.31) } \quad(4.13)^{* *} \quad(4.27)^{* *} \\
& \mathrm{R}^{-2}=0.38 ; \text { D.W. }=2.01 \\
& S_{t}=50.44+0.55 X_{t}-186.7 \text { Guerra }_{t} \\
& \text { (1.82) }(14.7)^{* *}(8.44)^{* *} \\
& \mathrm{R}^{-2}=0.88 ; \text { D.W. }=1.87 \\
& S_{t}=67.72-0.05 Y_{t}+63.9 \text { Guerra }_{t}+1.15 S_{l, t} \\
& \begin{array}{llll}
(1.28) \quad(1.45) & (1.27) & (9.16)^{* *}
\end{array} \\
& \mathrm{R}^{-2}=0.89 \text {. D.W. }=1.75 \\
& S_{t}=-29.19+0.64 X_{t}-220.7 \text { Guerra }_{t}-0.26 \text { So }_{t}
\end{aligned}
$$

La ecuación (45), es la estimación de la ecuación (41). Esto es la primera ecuación artificial. Aquí $\alpha_{1}$ es estadísticamente diferente de cero, con un nivel de confianza del $99 \%$, por ello puedo rechazar Ho. Esto sugiere que el PNB puede no ser la variable explicatoria adecuada para el ahorro. En el primer caso, ecuación (46), la 
cual es la ecuación artificial correspondiente a la ecuación (42), no puedo rechazar la hipótesis que el coeficiente $\alpha_{0}$ es igual a cero. Por ende esto sugiere que el conjunto de regresiones contienen ingreso por exportaciones es más apropiado para explicar el comportamiento del ahorro.

De la ecuación (44), la propensión marginal a ahorrar del ingreso del sector exportador es igual a 0.553 , con un nivel de significación de $1 \%$. El coeficiente de la variable aleatoria para la Guerra, nos sugiere que hubo un descenso en la función de ahorro, y es significativo a un nivel de $1 \%$. La constante tiene signo negativo, con un nivel de significancia de $8 \%$ revelando que la propensión media a ahorrar es menor que la propensión marginal a ahorrar. El R ajustado es 0.888 . $Y$ la prueba $F$ convencional 131.38 indica que toda la ecuación es estadísticamente significativa a un nivel de $1 \%$.

Una segunda forma de verificar si este procedimiento es adecuado o no es mediante la prueba Wald, imponiendo una restricción al coeficiente que acompaña el PNB en la versión ampliada de la función de ahorro propuesta por Maizels (1968):

$$
S_{t}=g+h Y_{t}+i X_{t}+j \text { Guerra }_{t}+V_{t}
$$

Dado que nuestro objetivo es conocer si el ahorro proviene principalmente del ingreso del sector exportador, nuestra hipótesis nula es que $h=0$. Las estimaciones de las ecuaciones con y sin restricción son:

$$
\begin{aligned}
\mathrm{St}= & -50.44+0.55 \mathrm{Xt}-186.7 \text { Guerra } \mathrm{t} \\
& (1.82) \quad(14.7)^{* *}(8.44) \\
\mathrm{R}^{-2}= & 0.88 ; \text { D.W. }=1.87
\end{aligned}
$$$$
\text { St }=9.47-0.05 Y t+0.639 X t-151.6 \text { Guerrat }
$$
(0.19)
$(9.16)^{* *}$
$(4.66)^{* *}$

$$
\mathrm{R}^{-2}=0.89 ; \text { D.W. }=1.75
$$


La ecuación (48), es la misma que la ecuación (44). La prueba Wald da una f estadística estimada de 2.09858 y los valores críticos para $F(1.30)$ son 4.17 y 7.56 , con niveles de significación de $5 \%$ y $1 \%$ respectivamente. Sin embargo no rechazan la hipótesis que el coeficiente Yt es igual a cero. Como hice ver en el capítulo anterior, esta puede ser considerada como una prueba que favorece el predominio de la brecha de ahorro durante todo el período, cuando la ecuación (16) representa ahorros ex-ante. Esto también es posible debido a que las exportaciones son mucho menores que el PNB, y la capacidad para ahorrar es restringida en este modelo por el ingreso proveniente de las exportaciones.

El ejemplo anterior, es la manera más simple de estimar la función de ahorro. Va más allá de la economía básica en el sentido de que se esta proponiendo que la principal variable explicatoria de la función de ahorro en El Salvador no es el PNB, si no más bien el ingreso proveniente del sector exportador. En lo que sigue, haré el mismo procedimiento con una teoría del ahorro diferente, en orden a ver si es posible encontrar un soporte adicional a los resultados anteriores.

Una teoría adicional del ahorro es la hipótesis del ingreso permanente propuesto por Milton Friedman. Establece que en el largo plazo los individuos buscan patrones estables de consumo y por ello no basan sus decisiones de ahorro o consumo en su ingreso presente, pero si en su ingreso esperado a lo largo de su vida, o riqueza dependiendo de su información actual. En este caso, la respuesta de los ahorros ante cambios en el ingreso permanente será nulo, mientras que la propensión marginal a ahorrar del ingreso transitorio. i.e. la diferencia entre el ingreso presente y permanente, estará cercana a uno.

La aplicación de la hipótesis del ingreso permanente es razonable en el caso de El Salvador en especial porque el ingreso de las exportaciones es bastante inestable, y de acuerdo a la hipótesis del ingreso permanente mientras más incierta es la fuente de ingreso, mayor es el efecto transitorio del ingreso en el nivel de ahorro. El conjunto de hipótesis a ser probadas siguiendo el procedimiento de la prueba J son: 
Ho: $S t=a+b Y P t+c Y T t+d G u e r r a t+U t$

$\mathrm{Hl}: \mathrm{St}=\mathrm{e}+\mathrm{FXPt}+\mathrm{gXTt}+\mathrm{hGuerra}+\mathrm{Vt}$

En donde, YTt y YTt, son los ingresos permanentes y transitorio basados en el PNB respectivamente, XPt $\mathrm{t} X \mathrm{XT}$ son los ingresos permanente y transitorio provenientes del sector exportador. Las funciones auxiliares son:

$$
\begin{aligned}
& \mathrm{St}=\mathrm{a}+\mathrm{bYPt}+\mathrm{cYTt}+\mathrm{dGuerra}+\alpha_{1} \mathrm{SHlt}+\mathrm{Ut} \\
& \mathrm{St}=\mathrm{e}+\mathrm{fXPt}+\mathrm{gXTt}+\text { hGuerra }+\alpha_{0} \mathrm{SHot}+\mathrm{Vt}
\end{aligned}
$$

En donde $\mathrm{SHo}$ y $\mathrm{SHl}$ son los valores predichos de las ecuaciones provenientes de $\mathrm{Ho}$ y $\mathrm{Hl}$. El ingreso permanente fue calculado como la media del ingreso actual y el ingreso en los dos períodos previos, mientras que el ingreso transitorio es la diferencia entre ingresos presente e ingreso permanente. Las dos funciones estimadas para el período 1960-1991 son:

Mientras $\alpha_{1}$ es estadísticamente diferente de cero $\alpha_{0}$ no lo es, entonces los resultados de la prueba J muestras que el conjunto de variables más apropiadas para explicar el comportamiento del ahorro, son el ingreso permanente y transitorio provenientes del sector exportador.

$$
\begin{aligned}
\mathrm{St}= & -236+0.26 \mathrm{YPt}-0.235 \mathrm{YtT}-417.6 \mathrm{Guerrat} \\
& (2.49)^{*} \quad(6.42)^{* *} \quad(1.04) \quad(6.55)^{* *} \\
\mathrm{R}^{-2}= & 0.64 \quad: \text { D.W. }=1.75 \\
\mathrm{St}= & -47.38+0.667 \mathrm{XPt}+0.607 \mathrm{XTt}-234.7 \text { Guerrat }-0.255 \mathrm{Hot} \\
& (1.45) \quad(6.91)^{* *} \quad(4.06)^{* *} \quad(4.57)^{* *} \\
\mathrm{R}^{-2}= & 0.88 ; \text { D.W. }=1.75
\end{aligned}
$$




$$
\begin{aligned}
\mathrm{St}= & 75.06-0.053 \mathrm{TPt}-0.026 \mathrm{Yt}+65.87 \text { Guerrat }+1.145 \mathrm{HIt} \\
& (1.12) \quad(1.14) \quad(0.19) \quad(0.92)(7.79)^{* *} \\
\mathrm{R}^{-2}= & 0.88 ; \text { D.W. }=1.82
\end{aligned}
$$$$
\text { St }=-58.5+0.56 \mathrm{XPt}+0.59 \mathrm{XTt}-180.9 \text { Guerrat }
$$$$
(1.84) \quad(12.97)^{* *}(3.93)^{* *} \quad(6.43)^{* *}
$$

Alternativamente, puedo utilizar la prueba $f$ en un modelo hibrido combinando la hipótesis de ingreso permanente y la idea de Maizels del ingreso separado del sector exportador.

$$
\mathrm{St}=\mathrm{a}+\mathrm{bYPT}+\mathrm{cYTt}+\mathrm{dXPt}+\mathrm{eXTt}+\mathrm{fGuerrat}+\mathrm{Vt}
$$

Aquí, yo impongo las restricciones que $b=0$ y $c=0$; luego estime la prueba Wald. Las ecuaciones con o sin restricciones estimadas son:

$$
\begin{aligned}
\mathrm{St}= & -58.5+0.56 \times \mathrm{Xt}+0.59 \times \mathrm{XT}-180.9 \text { Guerra } \mathrm{t} \\
& (1.84)(12.97)^{* *}(3.93)^{* *}(6.43)^{* *} \\
\mathrm{R}_{-2}= & 0.88: \text { D.W. }=1.82
\end{aligned}
$$

A pesar de que en la ecuación (58) estoy estimando seis parámetros

$\mathrm{St}=11.28-0.060 \mathrm{YPt}+0.0007 \mathrm{YTt}+656 \mathrm{XPt}+0.642 \mathrm{XTt}-137.58 \mathrm{Gt}$
(0.17)
(1.06)
(0.04)
$(6.09)^{* *}$
$(3.12)^{* *}$
$(2.47)^{*}$

$\mathrm{R}^{-2}=0.88 ;$ D.W. $=1.72$

Aun tengo 27 grados de libertad porque el tamaño de la muestra es suficientemente grande. La prueba $F$ para estas restricciones 
es 0.78005 y los valores críticos para $F(2,26)$ con niveles de significación de $1 \%$ son 5.53 y 3.37. Entonces no rechazaré la hipótesis nula que los coeficientes b y $c$ en la ecuación (43) son cero. Una vez más llego a la misma conclusión que en la prueba J, XPt y XTt por si solos explican mejor el comportamiento del ahorro.

\section{Capítulo III}

\section{Conclusiones}

Del capítulo anterior podemos derivar las siguientes conclusio nes:

1. El ejercicio de Landau para determinar la brecha dominante en el modelo de dos brechas, muestra que durante el período 19581991, hubo una situación de regímenes alternantes.

2. El método Chenery - Ekstein para pretender medir los dos brechas nos lleva a la conclusión que la brecha de ahorro fue dominante para 21 años de los 34 observados, sugiriendo que el ahorro nacional ha estado restringiendo la inversión, más que la escasez de divisa, especialmente en los setentas y ochentas.

3. La aplicación de la prueba Wald, imponiendo la restricción que los parámetros relacionados con el PNB son igual a cero, en el modelo Maizels Lee y el modelo combinado de la hipótesis de ingreso permanente apoyan la idea de que las exportaciones son más importantes para explicar el comportamiento del ahorro en El Salvador. Y de acuerdo al modelo de dos brechas observando la ecuación (16), la prueba también proporciona evidencia a favor del predominio de la brecha de ahorro durante el período estimado.

4. La prueba J para dos funciones de ahorro diferentes función Keynesiana simple y la hipótesis de ingreso permanente, indican que el conjunto conteniendo ingreso del sector exportador es más relevante para el que contiene PNB. Esto una vez más provee una manera indirecta de mostrar que la fuente más importante de ahorro en El Salvador es el ingreso generado por el sector exportador.

5. Los bajos niveles de ahorro nacional que conducen a un predo- 
minio de la brecha interna durante varios años, pueden estar vinculados al hecho que el modelo esta restringido a generar ahorros únicamente del ingreso exportador, que es mucho más pequeño que el PNB. Y también puede estar relaciona al hecho que los exportadores pueden ser un grupo con una alta propensión a consumir reforzada por efectos demostración y manejo del tipo de cambio y de la política de intercambio extranjero.

6. Para efectos de política económica, el objetivo de incrementar el ahorro nacional, podrá lograrse más fácilmente a través de políticas especificas que traten de estimular el ahorro en los grupos asociados con el sector exportador en lugar de la aplicación de políticas orientadas en términos agregados.

7. En todos las estimaciones relevantes de la función ahorro, la variable aleatoria para la guerra fue significante, lo cual sugiere que esta tuvo un impacto negativo en el ahorro nacional.

8. Un estudio más detallado de la función ahorro en El Salvador, conteniendo más información acerca del ahorro por sectores y grupos de ingreso (basado en censos y encuentas de gastos e ingresos) podría brindar dar más luz acerca del comportamiento y las posibilidades para incrementar la tendencia al ahorro en otros sectores. Así mismo sería de utilidad estudiar más detalladamente los flujos de ahorro destinados al financiamiento de inversión en otros sectores aparte del sector exportador.

\section{Apéndice A}

Todos los datos de esta tesis fueron obtenidos de la manera si 1 guiente: las figuras fueron copiadas en miles de colones de las tablas del costo nacional, productos y balances de las cuentas de pagos de la revista mensual del BCR diversos números:

- Producto nacional bruto PNB.

- Consumo.

- Inversión.

- Exportación de bs y Ss.

- Importaciones de los bs y Ss. 


\section{Notas}

1. A lo largo de la tesis, a menos que se mencione de otro modo, la notación es como sigue: Y: Producto nacional bruto; S: ahorro nacional bruto; to: X: exportaciones; M: importaciones; F: ahorro extranjero; y I: inversión.

2. Ver abajo las críticas en la introducción del ahorro extranjero como una variable explicatoria en la función de ahorro.

3. Los valores t están representados en paréntesis, * indica un nivel de significación de 5\% y ** indican un nivel de significación de $1 \%$.

4. Este coeficiente no representa la PMgSy en esta ecuación.

5. El procedimiento Hatanaka puede ser aplicado cuando existe un problema de autocorrelación en los residuos, y hay una variable dependiente rezagada en el modelo. Ver Greene (1993).

6. Este comando corre la regresión original y de los residuos obtenidos un estimado para rho. Luego genera una nueva variable dependiente usando este estimado de la variable dependiente original, y lo corre contra las variables independientes originales.

Posterior a esto obtiene los nuevos valores residuales utilizando las variables independientes originales, y obtiene un nuevo estimado para tho. Este proceso es repetido hasta que converge al valor de rho. (Hall Jhonston; y Lilien (190) p. 5-9).

7. Los coeficientes en esta ecuación no son los mismos que en la ecuación (2) en el Capítulo I. Después de aplicar algebra simple puede mostrarse que la ecuación equivalente es:

$$
S t=c+d Y t+(e-d) X t
$$

Queda la pauta para comparar los coeficientes con la ecuación original. De e d $=$ i y $h=d$, podemos deducir que $h$ es la PMgS del ingreso del sector no exportador, y $\mathrm{i}=\mathrm{h}$ es la $\mathrm{PMgS}$ del ingreso del sector exportador. 